09.1

\title{
Исследование спектра излучения молекулы СаО в диапазоне длин волн 540-650 nm в экспериментах на ударной трубе
}

\author{
(c) Н.О. Безверхний, Т.А. Лапушкина, Н.А. Монахов, М.В. Петренко, С.А. Поняев \\ Физико-технический институт им. А.Ф. Иоффре РАН, Санкт-Петербург, Россия \\ E-mail: nikolaybezverhny@gmail.com
}

Поступило в Редакцию 20 июля 2020г.

В окончательной редакции 30 сентября 2020 г.

Принято к публикации 2 октября 2020 г.

\begin{abstract}
Исследован спектр излучения молекулы $\mathrm{CaO}$ за фронтом отраженной ударной волны на ударной трубе диаметром $50 \mathrm{~mm}$. Спектры получены с помощью специально разработанного компактного спектрометра с CCD-линейкой, а также с помощью спектрометра на базе модернизированного монохроматора МДР-12 и фотоаппарата Sony SLT-A77 в качестве приемника излучения. Получены спектры излучения СаО при разных газовых температурах. Представленные результаты могут быть использованы для проверки теоретических моделей спектра излучения молекулы $\mathrm{CaO}$ при различных температурах.
\end{abstract}

Ключевые слова: ударная труба, отраженная ударная волна, оксид кальция.

DOI: 10.21883/PJTF.2021.02.50540.18473

Проблема движения тел в атмосфере Земли и планет Солнечной системы представляет значительный интерес как в случае летательных аппаратов, так и в случае космических тел, например метеоров. При входе в плотные слои атмосферы тело тормозится, в результате чего его поверхность испытывает нагрев, который приводит к интенсивной абляции. При движении в атмосфере крупных тел, таких как спускаемый аппарат и болид, с большой скоростью перенос тепла к обтекаемому телу потоками лучистой энергии доминирует над другими факторами нагрева его поверхности [1].

В работе [2] анализируется молекулярное излучение в спектре яркого болида абсолютной звездной величины -19.5. В спектрах были идентифицированы молекулярные полосы, принадлежащие оксидам $\mathrm{FeO}, \mathrm{CaO}, \mathrm{AlO}$ и $\mathrm{MgO}$. Проведено сравнение данных с теоретическими расчетами присутствия молекул в смеси метеорных паров и воздуха на различных высотах и при различных температурах. Однако $\mathrm{FeO}$ и $\mathrm{CaO}$ не были подробно проанализированы из-за того, что их молекулярные константы недостаточно определены. Молекулярные полосы $\mathrm{AlO}$ и $\mathrm{CaO}$ обнаруживаются методами лазерноискровой эмиссионной спектрометрии горных пород и отложений [3]. В то время как экспериментальному изучению спектра излучения молекулы $\mathrm{AlO}$ посвящено большое число работ, например [4,5], изучению спектра излучения молекулы $\mathrm{CaO}$ в оптическом диапазоне уделяется мало внимания. Двухатомная молекула $\mathrm{CaO}$ представляет интерес для астрофизических исследований [6,7]. Также предполагается, что $\mathrm{CaO}$, который диссоциирует на Са и О, является источником Са в атмосфере Меркурия [8]. Спектр молекулы $\mathrm{CaO}$ в видимом диапазоне длин волн наблюдался в экспериментах по взаимодействию быстролетящих медных снарядов с поликристаллическими доломитовыми мишенями [9]. По- пытки моделирования спектра излучения молекулы $\mathrm{CaO}$ проводились в работе [10]. Авторы провели моделирование спектра излучения $\mathrm{CaO}$ в области 590-630 nm, которое сравнивалось с экспериментально наблюдаемыми спектрами болида. Было достигнуто умеренное согласие модели с экспериментальными спектрами. Наибольшая неопределенность при моделировании была связана с наличием в спектре полос $\mathrm{FeO}$, которые перекрываются с полосами $\mathrm{CaO}$ в области 590-630 nm.

В настоящей работе продемонстрирована возможность исследования спектра молекулы $\mathrm{CaO}$ в оптическом диапазоне длин волн в газодинамическом эксперименте на ударной трубе. В спектрах не наблюдается полос $\mathrm{FeO}$, что дает возможность независимо исследовать спектр излучения $\mathrm{CaO}$ в области 590-630 nm.

Схема экспериментальной установки представлена на рис. 1. В ее основе лежит ударная труба, состоящая из камеры высокого давления (КВД) 14 и камеры низкого давления (КНД) 12. Ударная труба непосредственно соединена с измерительной секцией 5 с торцом из сплава алюминия 6 , а затем через диафрагменный блок 7 с демпферным баком 8 .

КВД 14 представляет собой канал круглого сечения длиной $100 \mathrm{~cm}$ и диаметром $5 \mathrm{~cm}$ для напуска толкающего газа. Перед экспериментом КВД откачивается безмасляным форвакуумным насосом до давления $10 \mathrm{~Pa}$, затем заполняется толкающим газом до половины рабочего давления.

КНД 12 - это труба круглого сечения длиной $432.5 \mathrm{~cm}$ и диаметром $5 \mathrm{~cm}$, которая заполняется рабочим газом. Камера откачивается форвакуумным насосом. Контроль вакуума и рабочего давления производится с помощью датчика Пирани InstruTech CVG101GC. Камера откачивается до вакуума $0.1 \mathrm{~Pa}$, затем заполняется 


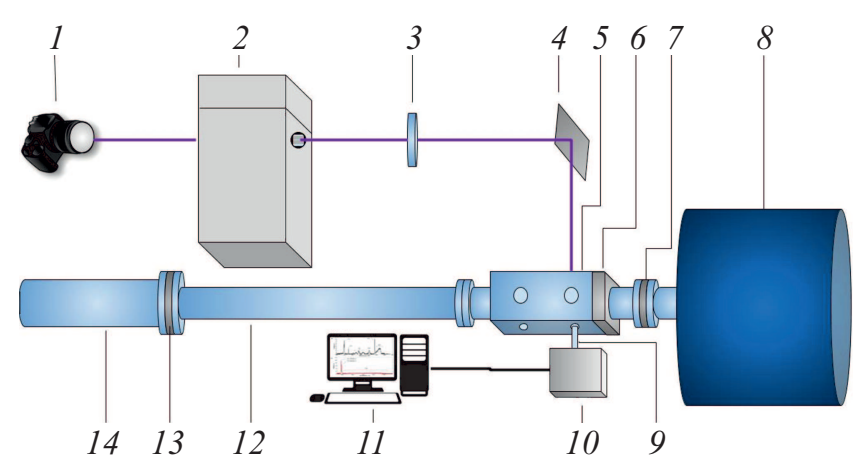

Рис. 1. Схема установки. 1 - фотоаппарат Sony SLT-A77, 2 - МДР-12, 3 - линза, 4 - зеркало, 5 - измерительная секция, 6 - торец, 7,13- диафрагменные блоки, 8 - демпферный бак, $9-$ коллиматор, $10-$ компактный спектрограф, 11 - ПК, 12 - камера низкого давления, 14 - камера высокого давления.

рабочим газом; диапазон рабочих давлений от 130 до $10^{5} \mathrm{~Pa}$.

КНД от КВД отделяется алюминиевой диафрагмой, расположенной в диафрагменном блоке 13 между камерами. Перед диафрагмой установлен крестообразный нож, который способствует разрыву диафрагмы. После разрыва диафрагмы в ударной трубе формируется падающая ударная волна. При отражении падающей ударной волны от торца 6 происходит нагрев газа за счет перехода кинетической энергии газового потока во внутреннюю энергию.

Для измерения скорости ударной волны в измерительной секции 5 на расстоянии $52 \mathrm{~mm}$ друг от друга размещены пьезоэлектрические датчики давления. Сигнал с датчиков давления поступает на четырехканальный цифровой осциллограф (TDS-2024C), подключенный к компьютеру. Длительность временно́й развертки осциллографа в данных экспериментах составляла $100 \mu \mathrm{s}$, шаг временно́го интервала - $0.04 \mu \mathrm{s}$. Погрешность измерения скорости ударной волны не превышает 5\%. Измерительная секция оборудована кварцевыми окнами, через которые осуществляются спектральные измерения. Интенсивности атомных линий и молекулярных полос регистрировались с помощью компактного спектрографа 10 со спектральным разрешением $\sim 0.7 \mathrm{~nm}$, а также монохроматора МДР-12 (2 на рис. 1), модернизированного для работы в качестве спектрографа. Для этого с МДР-12 была снята выходная щель. Фотоаппарат Sony SLT-A77 (1 на рис. 1) с макрообъективом Sony $100 \mathrm{~mm} \mathrm{f} / 2.8$ Масro фокусировался на плоскость выходной щели. Спектральное разрешение такой системы составляет $\sim 0.3 \mathrm{~nm}$. В экспериментах измеряются интегральные по времени спектры излучения газа. Калибровка по длинам волн проводилась с помощью ртутной лампы, калибровка спектральной чувствительности - с помощью калиброванной лампы накаливания ТРШ 2850-3000.
В настоящей работе в качестве рабочего газа использовался воздух, в качестве толкающего газа водород. На рис. 2, $a$ и $b$ представлены полученные в результате обработки спектры при давлении воздуха в КНД $P_{1}=0.93 \pm 0.09 \mathrm{kPa}$, давление водорода в КВД, при котором произошел разрыв диафрагмы, $P_{4}=4.25 \pm 0.01 \mathrm{MPa}$. Скорость ударной волны, измеренная по сигналам датчиков давлений в измерительной секции, составила $V=2.9 \pm 0.2 \mathrm{~km} / \mathrm{s}$, что соответствует числу Маха $\mathrm{M}=8.7 \pm 0.3$.

Идентификация наблюдаемых в спектрах атомарных линий осуществлялась с помощью базы данных спектральных линий NIST Atomic Spectra Database. Линии принадлежат металлам $\mathrm{Al}, \mathrm{Ca}$, Na. Также в спектре наблюдаются молекулярные полосы $\mathrm{AlO}\left(B^{2} \Sigma^{+}-X^{2} \Sigma^{+}\right)$, которые были идентифицированы по работам [4,5], и полосы излучения молекул $\mathrm{CaO}$ переходов $\left(d^{3} \Delta_{2}-a^{3} \Pi_{1}\right)$, $\left(e^{3} \Sigma-a^{3} \Pi_{1}\right),\left(c^{3} \Sigma^{+}-a^{3} \Pi_{1}\right),\left(C^{1} \Sigma^{+}-a^{3} \Pi_{1}\right),\left(D^{1} \Delta-a^{1} \Pi_{1}\right)$, $\left(c^{3} \Sigma_{1}-a^{3} \Pi_{0}\right), \quad\left(c^{3} \Sigma^{+}-a^{3} \Pi_{2}\right)$ и др. [9]. Наблюдаемые в спектре атомарные линии и молекулярные полосы являются следствием взрывного испарения вещества с поверхности торца при торможении ударной волны. Торец измерительной секции изготовлен из сплава алюминия (марки АМг), что объясняет наличие в спектре линий излучения атомов $\mathrm{Al}, \mathrm{Ca}$ и $\mathrm{Na}$, а также полос $\mathrm{AlO}$ и $\mathrm{CaO}$. Молекулы $\mathrm{CaO}$ и $\mathrm{AlO}$ также могут образовывать-
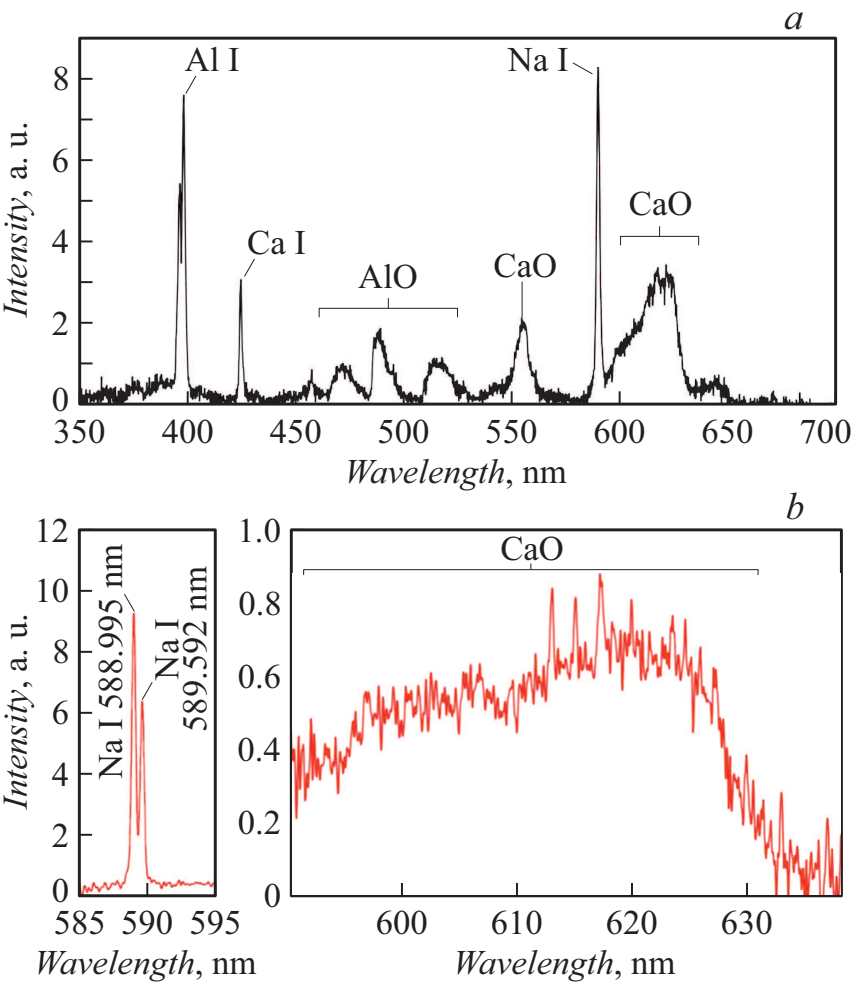

Рис. 2. Спектры излучения отраженного от торца газа. $a$ - спектр, полученный на компактном спектрометре; $b$ - спектр, полученный при помощи спектрометра на базе монохроматора МДР-12. 


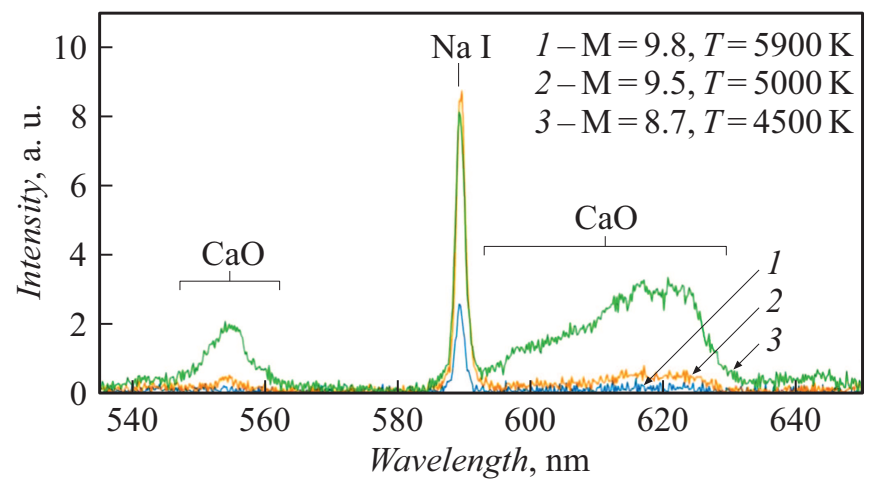

Рис. 3. Спектры излучения отраженного от торца газа для различных значений числа Маха.

Результат обработки экспериментальных данных

\begin{tabular}{c|c|c|c}
\hline$P_{1}, \mathrm{kPa}$ & $P_{4}, \mathrm{MPa}$ & $\mathrm{M}$ & $T, \mathrm{~K}$ \\
\hline $0.40 \pm 0.04$ & $4.4 \pm 0.1$ & $9.8 \pm 0.5$ & $5900 \pm 600$ \\
$0.53 \pm 0.05$ & $4.4 \pm 0.1$ & $9.5 \pm 0.5$ & $5000 \pm 250$ \\
$0.93 \pm 0.09$ & $4.3 \pm 0.1$ & $8.7 \pm 0.4$ & $4500 \pm 130$
\end{tabular}

ся в результате реакций между атомами на поверхности торца и кислородом, содержащимся в воздухе.

Для определения температуры проводилось сравнение экспериментальных данных с моделью спектра излучения $\mathrm{AlO}\left(B^{2} \Sigma^{+}-X^{2} \Sigma^{+}\right)$, построенной на основании работы [11]. Моделирование проводилось в предположении, что излучающая плазма находится в тепловом равновесии. Наилучшее соответствие между экспериментальными данными для числа Маха $\mathrm{M}=8.7$ и моделью наблюдается при температуре $T=4500 \mathrm{~K}$.

В таблице приведены значения температуры газа для различных режимов, определенные по молекулярным полосам $\mathrm{AlO}$, значения чисел Маха и начального давления газа. Данные получены путем обработки соответствующих спектров излучения, приведенных на рис. 3 . Из рисунка видно, что тенденция изменения интенсивности излучения полос $\mathrm{CaO}$ отличается от поведения интенсивности атомарных линий, в частности, атома Na. Это отличие можно объяснить разной скоростью возбуждения соответствующих энергетических уровней у атомов и молекул вследствие различия механизмов энергетического обмена, а также нагрева молекулярных и атомарных газов [12]. Быстрая ответная реакция спектра полос молекулы $\mathrm{CaO}$ на изменение температуры газа позволяет использовать его для определения температуры газа, в том числе для быстропротекающих газодинамических процессов. Для уточнения методики требуются теоретические расчеты спектра излучения $\mathrm{CaO}$ в присутствии молекул $\mathrm{AlO}$ и атомов $\mathrm{Na}, \mathrm{Ca}$ и $\mathrm{Al}$.

Таким образом, в работе получены спектры излучения молекулы $\mathrm{CaO}$ за фронтом отраженной ударной волны в экспериментах на ударной трубе. Проведена оценка температуры по наблюдаемым в спектре молекулярным полосам AlO. Продемонстрирована возможность исследования спектра молекулы $\mathrm{CaO}$ в оптическом диапазоне в газодинамическом эксперименте на ударной трубе. Полученные экспериментальные данные могут быть использованы для проверки теоретических моделей спектра излучения молекулы $\mathrm{CaO}$ при различных температурах.

\section{Конфликт интересов}

Авторы заявляют, что у них нет конфликта интересов.

\section{Список литературы}

[1] С.T. Суржиков, Радиационная газовая динамика спускаемых космических аппаратов. Многотемпературные модели (Институт проблем механики им. А.Ю. Ишлинского РАН, М., 2013).

[2] J. Borovička, A.A. Berezhnoy, Icarus, 278, 248 (2016).

[3] N. Killiny, E. Etxeberria, A.P. Flores, P.G. Blanco, T.F. Reyes, L.P. Cabrera, Sci. Rep., 9, 2449 (2019).

[4] J. Hermann, A. Lorusso, A.Perrone, F. Strafella, C. Dutouquet, B.Torralba, Phys. Rev. E, 92, 053103 (2015).

[5] J.M. Peuker, P. Lynch, H. Krier, N. Glumac, Propellants Explos. Pyrotech., 38, 577 (2013).

[6] S. Sakamoto, G.J. White, K. Kawaguchi, M. Ohishi, K.S. Usuda, T. Hasegawa, Mon. Not. Roy. Astron. Soc., 301, 872 (1998).

[7] E.W. Guenther, J. Cabrera, A. Erikson, M. Fridlund, H. Lammer, A. Mura, H. Rauer, J. Schneider, M. Tulej, Ph. von Paris, P. Wurz, Astron. Astrophys., 525, A24 (2011).

[8] R.M. Killen, Icarus, 268, 32 (2016).

[9] S. Sugita, P.H. Schultz, S. Hasegawa, JGR: Planets, 108, 5140 (2003).

[10] A.A. Berezhnoy, J. Borovička, J. Santos, J.F. Rivas-Silva, L. Sandoval, A.V. Stolyarov, A. Palma, Planet. Space Sci., 151, 27 (2018).

[11] А.В. Ефимов, Развитие методов комплексного спектрального анализа многокомпонентной движущейся плазмы импульсных разрядов, канд. дис. (Объединенный институт высоких температур РАН, М., 2017).

[12] D. Staack, B. Farouk, A. Gutsol, A. Fridman, Plasma Sources Sci. Technol., 17, 025013 (2008). 